\title{
Curtain Airbag design for Robust Deployment
}

\author{
Claudio Guerra, Nicola Leomanni
}

\begin{abstract}
When Curtain Side Airbags are validated in real environment, a great variability in deployment behavior and performance is observed and many optimization loops are executed to mitigate that variability, with potential extra costs and time delays. The present study analyzes the Curtain Airbag performance in order to develop design best practices able to reduce the variability of his behavior and maximize the performance of his deployment. To achieve this goal the DFSS methodology has been applied and ad hoc experimental parameters have been identified to evaluate Curtain Airbag behavior. Noise factors and control factors have been defined and experimental testing has been executed to evaluate the contribution of each control factor to the overall performance. Finally, control factors that maximize the Signal to Noise ratio and the airbag performance have been chosen and translated into engineering best practices.
\end{abstract}

Index Terms - SABIC; DFSS; Robust Engineering; Airbag Deployment

\section{INTRODUCTION}

Curtain Airbag, commonly known as Side Airbag Inflatable Curtain (SABIC), is an airbag which deploys on each side of the vehicle in order to prevent fatal injuries to the occupant head, particularly for lateral impact accidents.

In real life environment the SABIC has to properly deploy inside a complete vehicle system, interacting with a high number of components (i.e. headliner, ramp brackets, trims, absorbers). The interaction with surrounding parts, with their own tolerances, may have influence on bag deployment and trim integrity and at the end, on customer protection. Moreover, the SABIC has to maintain a stable response and positioning with different environment conditions (such as temperature), wearing and aging status.

Usually, intensive hardware tests have to be conducted to ensure clean deployment of the curtain airbag through trim when the prototype trims parts are available. [1]

All these factors contribute to make the development process of a SABIC for a new vehicle very complex and sensible to late changes in the design, also due to the fact that final design freeze is possible only with the availability of vehicles with a status very close to final production.

Manuscript revised on July 02, 2019 and published on August 10, 2019 Claudio Guerra, Emea Product Development, FCA Italy, Email: claudio.guerra@fcagroup.com ${ }^{1}$ nicola.leomanni@fcagroup.com ${ }^{2}$

Nicola Leomanni, Emea Product Development, FCA Ital. Email: nicola.leomanni@fcagroup.com ${ }^{2}$

\section{OBJECTIVES}

It has been applied a "DFSS" methodology with the following target: develop robust design best practices that permit to reduce variability of airbag deployment behavior, minimizing the sensibility of the airbag to external factors (noises).

Robustness in DFSS is defined as "the state of performance where the technology, product, or process is minimally sensitive to factors causing variability at the lowest possible cost." [2]

\section{DFSS METHOD}

Dr. Taguchi's DFSS method follows the IDDOV Identify, Define, Develop, Optimize, and Verify - process. In this paper the focus is on the Optimize phase. The purpose of the Optimize Phase is to develop the product to be robust.

Robust optimization uses orthogonal arrays to conduct efficient experiments that are balanced. [2] The key of the Optimize Phase is the parameter design process. In the parameter design the aim is to measure and optimize the variability and efficiency of the energy transformation of the system in consideration. [4]

\section{PARAMETERS IN SCOPE OF THE PROJECT}

Only parameters that are directly related to cushion design are within the scope of the activity; all the others such as inflator performances, interiors trims designs and Body-in-white design are considered out of scope.

\section{TESTING PROCEDURE AND IDEAL FUNCTION}

In order evaluate the performances of the SABIC the vehicle has been virtually divided in a number of different vertical paths. For each paths, the amount of vertical displacement of the cushion during time is the function that describes the system performance.

The ideal function for the system is linear:

\author{
$\mathbf{Y}$ (output response) $=\beta \times \mathbf{M}$ (input signal) \\ $\mathrm{Y}=\%$ of cushion displacement \\ $\mathrm{M}=$ time (ms) \\ $\mathrm{B}=$ slope of the output response.
}

To get a plot of the function in the real environment through the analysis of SABIC deployment videos, the percentage of SABIC deployment has been recorded at $1 \mathrm{~ms}$ step, describing the speed of the vertical cushion displacement in each section of the vehicle. 
To evaluate the percentage of SABIC deployment along each specific path, the amount of cushion deployed in the video frame has been measured by software, setting the zero at the headliner lower profile (before airbag activation). Then this amount of cushion deployed has been compared to the total amount (airbag fully deployed) to get the percentage for each step, see fig. 1 .

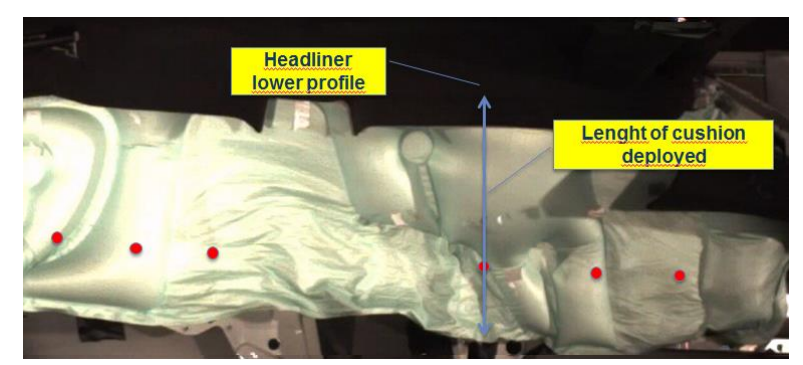

Figure 1. Deployed cushion measurement

\section{PRELIMINARY TESTING ON CURRENT SCENARIO}

Preliminary tests on current production vehicle have been executed.

The outcome of the testing was useful also to validate measurement procedure for reproducibility and repeatability.

For the testing, a B-segment SUV vehicle and n.10 different paths have been chosen. In order to have a visible reference, the Body in white has been marked with properly spaced red dots. These references have been used to define the vertical paths in the position judged as more valuable, see next fig 2 .

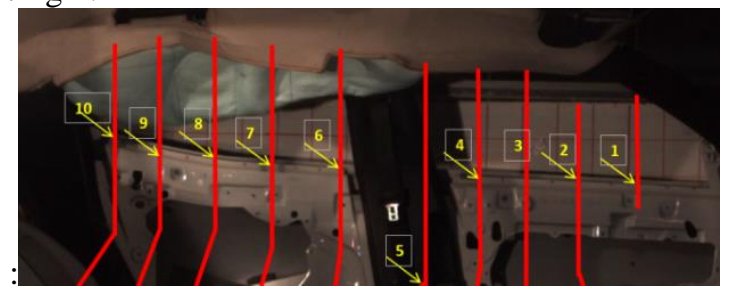

Figure 2. Vertical paths definition

Some static SABIC deployments tests has been recorded and the typical result is plotted in fig. 3 .

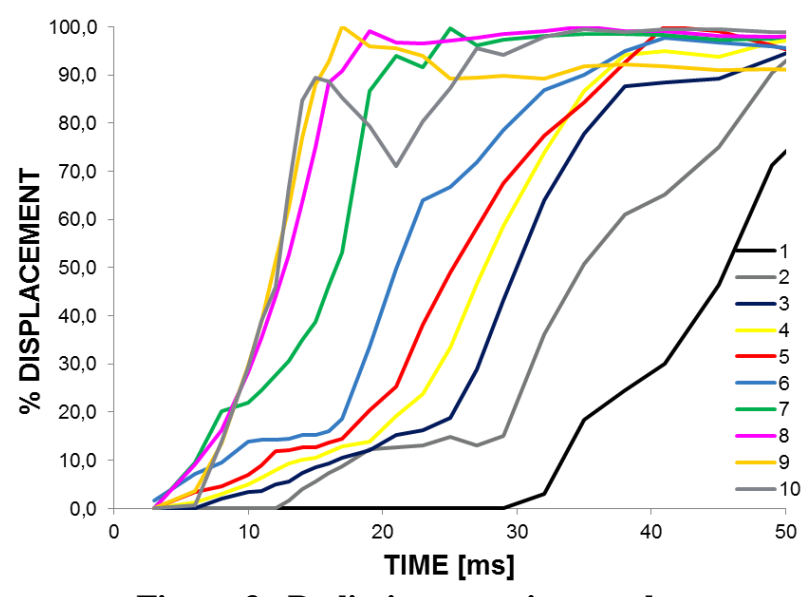

Figure 3. Preliminary testing results
It is evident that the rear paths (from n.10 to n.7) are significantly faster than the average.

Meanwhile the front paths (from n.3 to n.1) are significantly slower than the average.

This indicated that the coverage speed of the airbag cushion is not balanced, creating areas in which the cushion deployed in advance respect to other areas, forcing the engineers to increase gas generator output to reach proper cushion coverage also in the slower areas.

\section{SET UP FOR DEFINITIVE TESTING}

In order to manage and to optimize the big amount of experimental data it has been decided focus only on the portion of cushion that really influences the safety performance: the first impact regions of the occupant's head on side crash.

Referring to the $n .10$ paths of the preliminary testing the important paths to be recorded are n. 4,5 and 9,10. See fig. 4 .
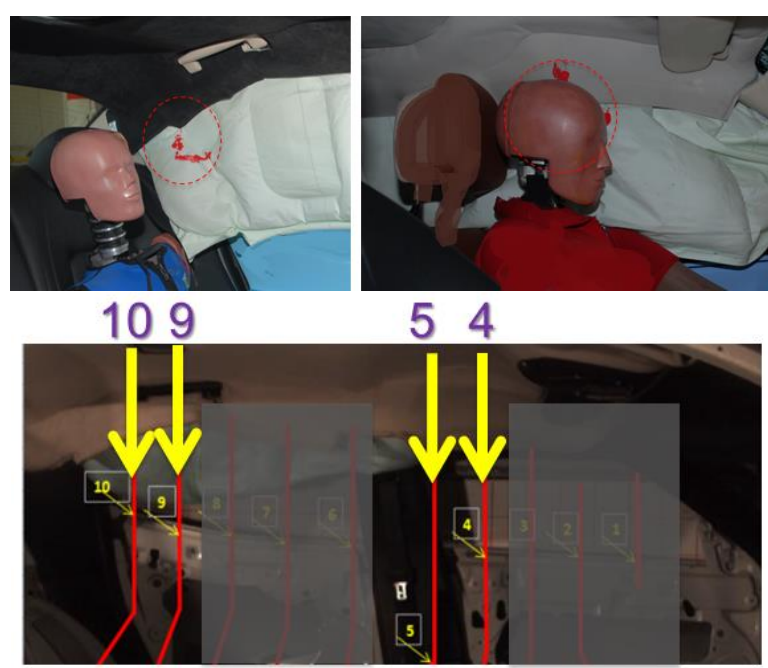

Figure 4. Paths correlated with head impact position
According to DFSS methodology, these are the factors that are outside the control of the designer, due to either practical or economic reasons, such as environmental condition or aging of materials. [3]

All "noise" effects produced by real environmental are very difficult to be reproduced in numerical simulations, particularly in the initial phase of an airbag development. Creating an accurate model of a folded airbag is a challenge, even with the latest improvements of the corpuscular particle method (CPM) [1]. Therefore, standard design methodology is based on previous lessons learned without a systematic approach and without knowing which design feature is really affecting robustness of the deployment. Consequently, many modifications in late project phase can occur, even close to the vehicle launch.

Noise factors are chosen that will likely cause the system to perform at lower levels of performance. The noise strategy is to compound these factors together to introduce maximum variation.

For each noise factor, two levels (N1 and N2) were 
defined: with a LOW (N1) and a HIGH (N2) effect on system output response.

Low and High effects have been identified by engineering experience or in case of doubt with dedicated experiments.

Noise factors taken in consideration are:

A) Headliner overlapping in the trim pillars:

This factor represents the variability in the tolerance of overlapping between headliner edge and pillars trim edge, see fig.5:

$\mathrm{N} 1$ : maximum overlapping $(10 \mathrm{~mm})$

$\mathrm{N} 2$ : minimum overlapping $(6 \mathrm{~mm})$
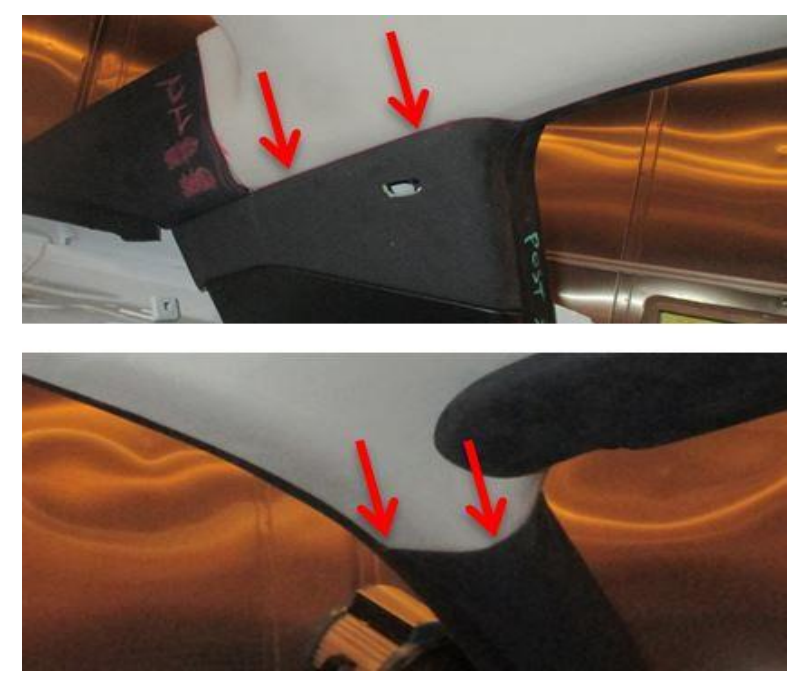

Figure 5. Overlapping between Headliner and trims

B) Aging condition of the SABIC:

N1: aged condition simulating 15 years life.

N2: new condition;

C) Cushion positioning under trim:

This factor represents the variability in the distance between Body in White (B.I.W.) and cushion pack in rest position (see fig. 6):

$\mathrm{N} 1: 10 \mathrm{~mm}$ distance.

$\mathrm{N} 2$ : cushion package in contact to body in white

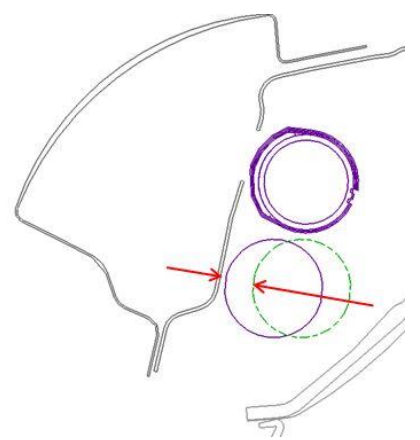

Figure 6. Distance between cushion pack and B.I.W.

D) Number of clips for A pillar Trim fixation.

This factor represents the variability in typical design for the number of retention point for the A pillar trim (see fig. 7):
N1: 3 clips.

N2: 2 clips.

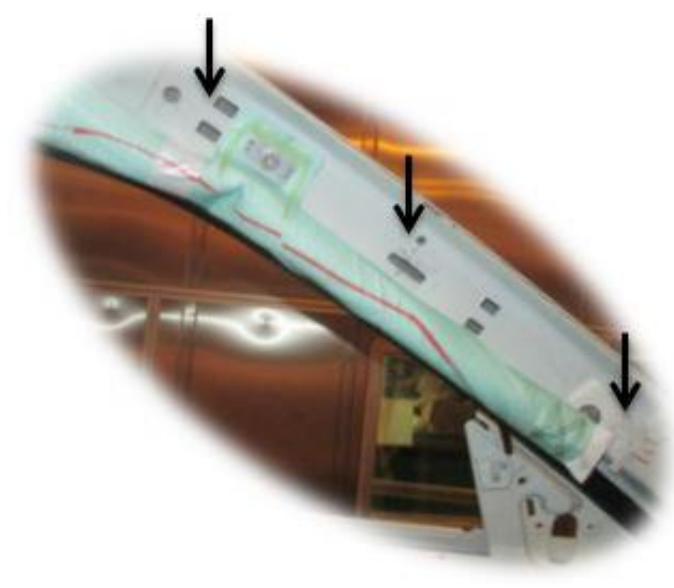

Figure 7. A - Pillar clips positions

E) Temperature of environment during airbag deployment:

$\mathrm{N} 1$ : cold condition $\left(-20^{\circ} \mathrm{C}\right)$

$\mathrm{N} 2$ : room temperature $\left(23^{\circ} \mathrm{C}\right)$;

\section{CONTROL FACTORS}

Control factors are those design and process factors that can be selected and fixed to a certain level after Parameter Design. [3]

For our purpose control factors are the parameters that can be used in airbag design in order to optimize the deployment, and they are defined with precise levels.

In the control factor description, dimensions have not been indicated, due to company intellectual property.

The Control Factors taken in consideration are:

\section{A) Cushion folding:}

The basics cushion folding used for the SABIC are zig-zag and roll folding. With zig-zag folding the cushion is folded in an accordion pattern, in the roll folding the cushion is rolled on itself. With combination of this two basic folding patterns is possible to create many different types of cushion folding. Here six types are evaluated, corresponding to following levels:

A1: Roll outboard

A2: n. 1 Vertical zig zag + roll

A3: n2 Vertical zig zag + roll

A4: Roll inboard

A5: n1 Horizontal zigzag + roll

A6: $n 2$ Horizontal zigzag + roll

B) Internal diffuser central opening geometry:

The internal gas diffuser is a fabric structure sewed inside 
the cushion that can deflect the exit gas from Inflator in predefined directions through lateral and central openings.

Three types of diffuser central openings are evaluated, corresponding to following levels (see fig.8)

\section{B1: No opening \\ B2: small opening \\ B3: big opening}

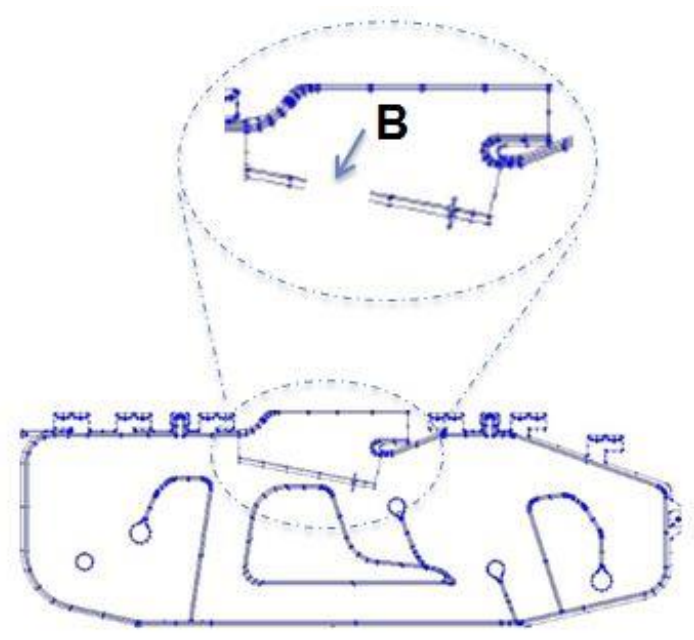

Figure 8. Diffuser central opening

C) Internal diffuser lateral openings, see fig. 9.

Three types of diffuser lateral openings are evaluated, corresponding to following levels (see fig..)

$\mathrm{C} 1$ : same size openings

$\mathrm{C} 2$ : ratio $>1$ (front diffuser opening bigger than rear)

$\mathrm{C} 3$ : ratio $<1$ (front diffuser opening smaller than rear)

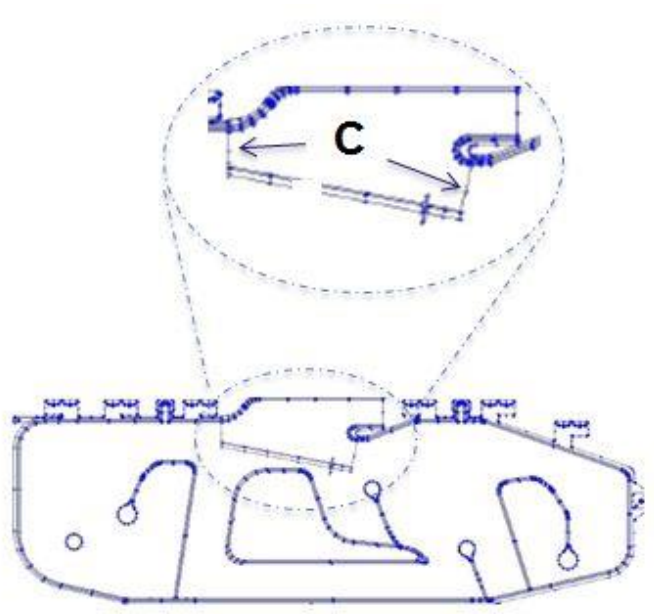

Figure 9. Diffuser lateral openings

Control factors D, E and F: are related to cushion shape and design:

D) bottom horizontal channel connecting front camera with rear camera:

Three types of horizontal channels are evaluated, corresponding to following levels (see fig10)

D1: no channel
D2: small channel

D3: large channel

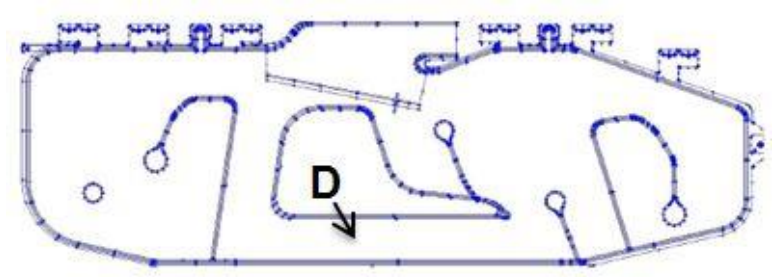

Figure 10. Horizontal channel

E) Front cushion design

Three types of front designs are evaluated, corresponding to following levels (see fig11):

E1: without delayed chamber

E2: with delayed chamber

E3: with "twister" shaped chamber

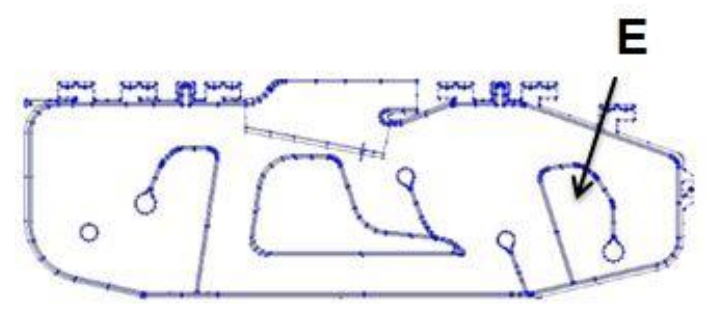

Figure 11. Front cushion design

F) Rear cushion design

Three types of rear designs are evaluated, corresponding to following levels (see fig12):

F1: with delayed chamber

F2: without delayed chamber

F3: with "twister" shaped chamber

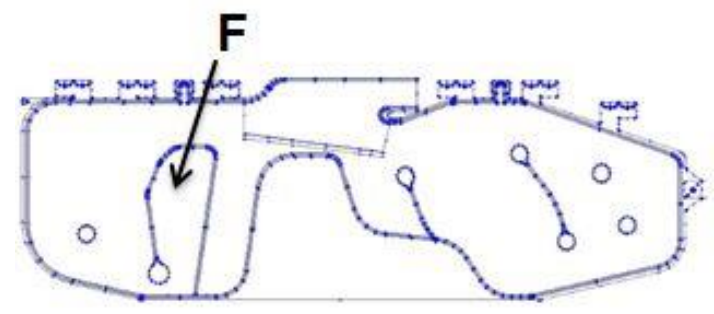

Figure 12. Rear cushion design

G) Additional rear folding:

Folding around a vertical line on the rear chamber executed before or after the complete folding of the cushion:

Three types of rear folding designs are evaluated, corresponding to following levels (see fig11):

G1: Prefold

G2: None

G3: Postfold 


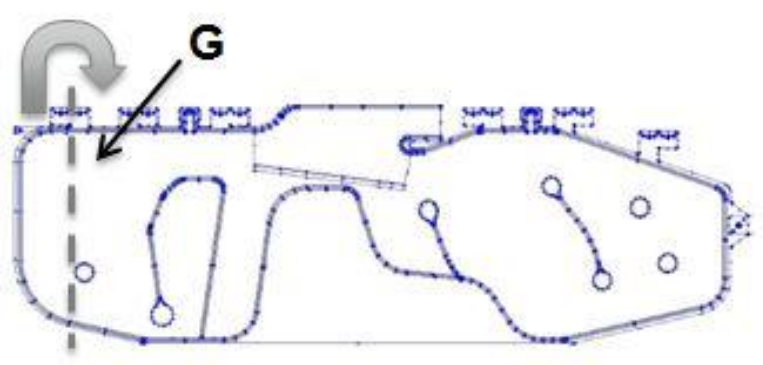

Figure 13. Rear pre/post folding

\section{TESTING EXECUTION}

A proper orthogonal array is selected according to how many different control factors and levels are needed for the experiments. In this case an L18 approach has been used with n.1 six levels control factor and n.6 three levels control factors, see table1. Each row of the array represents 2 experiment with the same control factors but with different noise configuration: one with noises in configuration $\mathrm{N} 1$ and one in configuration $\mathrm{N} 2$.

In each noise configuration each vertical line is related to the output (\% of cushion deployment) related to a specific video frame timing. The step used is $3 \mathrm{~ms}$.

The procedure is repeated for each of the four vehicle's sections considered for evaluating the performance (n. 4,5 and 9,10$)$.

\section{Table 1}

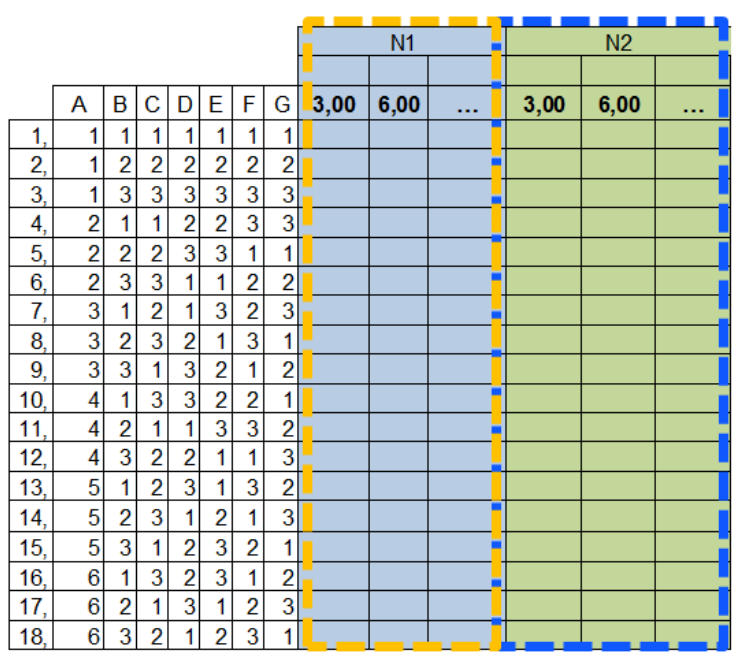

For each experiment an overall $\mathrm{S} / \mathrm{N}$ and a $\beta$ is calculated, taking in account all the outputs (outputs of each time frame and each path). The $\mathrm{S} / \mathrm{N}$ is an index of robustness. The higher the $\mathrm{S} / \mathrm{N}$, the more the system is doing what it is intended to do, being less sensitive to noise factors. It measures the quality of energy transformation that occurs within a design [2].

$\mathrm{S} / \mathrm{N}=$ useful output energy / harmful output energy[3]. The slope of the output response $(\beta)$ is the sensitivity of the input signal to the output response.

Response tables are created by calculating the $\mathrm{S} / \mathrm{N}$ and $(\beta)$ level averages for each control factor [3]. See fig. 14.

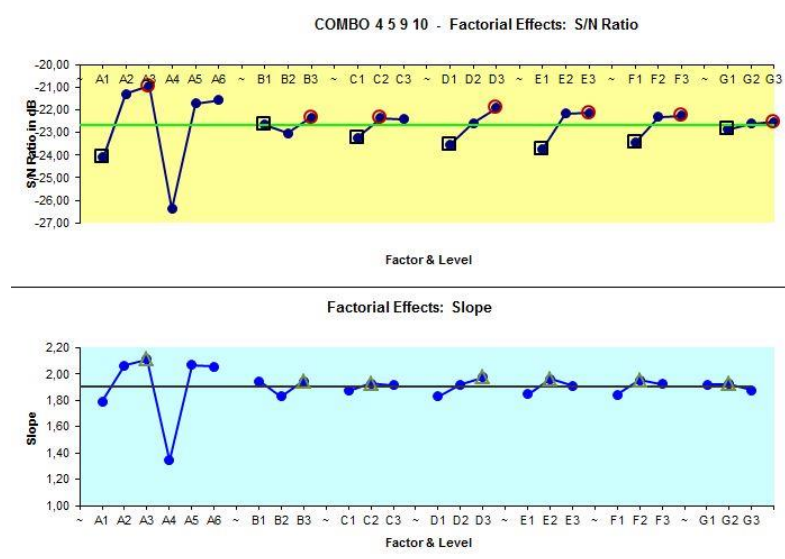

Figure 14. S/N and $\beta($ slope) response tables.

With the $\mathrm{S} / \mathrm{N}$ response table is possible to identify the control factor levels that maximize the $\mathrm{S} / \mathrm{N}$.

But together with the $\mathrm{S} / \mathrm{N}$ ratio we want to maximize also the performance of SABIC deployment, and that is related to the slope of the ideal function, so the control factor must be identified taking in consideration not only the effect on $\mathrm{S} / \mathrm{N}$ but also on $(\beta)$, see fig. 15 .

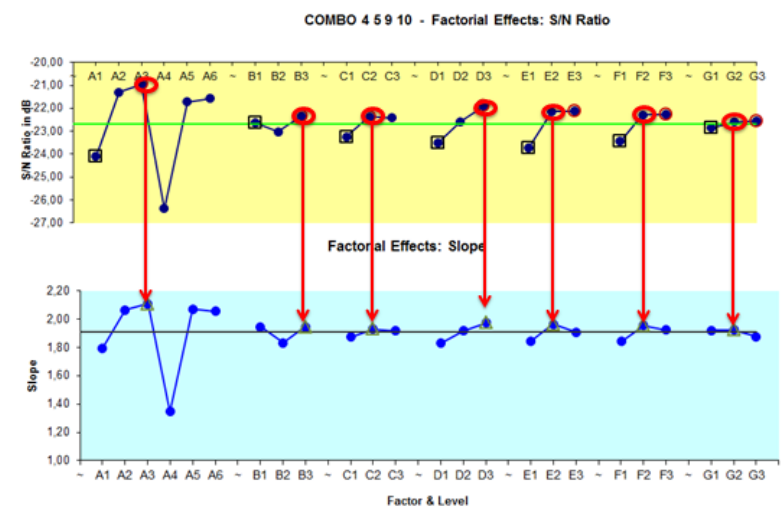

Figure 15. Control factors selection

The outcome of this selection of control factor's level is:

A3: Outboard roll+2vertzigzag

B3: diffuser center opening large size:

C2: diffuser: big opening towards the front

D3: large horizontal channel

E2: front delayed chamber

F2: w/o rear delayed chamber

G2: no rear post/pre fold

This selection of control factors permits to have the most robust configuration for SABIC deployment.

The signal to noise ratio and the $\beta$ of this configuration are: $\mathrm{S} / \mathrm{N}=-20.9 \mathrm{~dB} ; \beta=2.17$

The initial configuration of the system was:

A1: Outboard roll

B1: no diffuser center opening

C1: diffuser: same size lateral openings

D1: no horizontal channel 
E1: without front delayed chamber

F1: with rear delayed chamber

G1: with rear pre fold

The signal to noise ratio and the $\beta$ of this configuration are: $\mathrm{S} / \mathrm{N}=-31.4 \mathrm{~dB} ; \beta=1.03$

The achievements of the optimized configuration respect to the initial is: $+34 \%$ on $\mathrm{S} / \mathrm{N}$ and $+111 \%$ on $\beta$ : an important improvement either for robustness and for performances.

\section{CONCLUSIONS}

Current DFSS project proved that the airbag performance can be stabilized $(+34 \%)$ and fastened $(+111 \%)$ with the appropriate choose of design control factors. The most effective control factor is cushion folding. Front and rear chamber geometry, together with horizontal channel geometry are also important for optimization, meanwhile all the others control factors give small contribution.

These optimized control factors can be directly translated in general design guidelines. Using optimized guidelines is

\section{AUTHORS PROFILE}

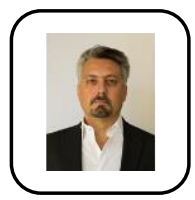

Claudio Guerra, Mechanical Engineer, M.D. at Politecnico di Turin, with 13years experience in passive safety engineering within Fiat Chrysler Automobiles and Maserati. He has been Italian Member in workgroup: CEN/TC212/WG4: ISO 14451 - Pyrotechnic articles for vehicles (2013). Currently is Design Responsible for Seat Belts and referent expert for Gas Generators technology within FCA Emea He is DFSS GreenBelt qualified, with two main internal released studies about Improved Steer. Wheel quality perception and Horn system optimization and several studies related to airbag performance.

$\mathrm{He}$ is also Reactive GreenBelt qualified for problem solving: main studies are related to IP door integrity with Passenger Airbag cold deployment, Horn activation system issue on Driver Airbag and Radio switches issue on last generation Steering wheel.

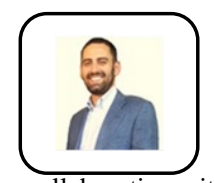

Second Author Nicola Leomanni, Mechanical Engineer, M.D. at Politecnico of Turin with also a Master Degree in Mechanical Engineering at the University of Illinois, Chicago (Graduation Date May 2006) with a monography on "Modeling And Identification Of Mistuned Blisks" in collaboration with Avio-GE

He had a short experience in Accenture (2006-2007) as on-site consultant for a spare parts innovation program.

He has +10years experience in passive safety design (2007 on) within Fiat Chrysler Automobiles Company and Maserati.

He has been design responsible for lateral airbags and he is currently responsible for the entire restraints perimeter in Fiat Chrysler Automobiles for the EMEA Region, including all airbags, seatbelts and steering wheel components.

He is Black Belt Design For Six Sigma (ASI Consulting Group certification) since July 2017: his most important internal studies relate to lateral airbag performance and reduced package designs. possible to achieve a robust response during first validation tests (static activations and crashes), reducing SABIC development timing and risks.

\section{REFERENCES}

[1] Feng, B., Coleman, D. "Gas Dynamic Simulation of Curtain Airbag Deployment through Interior Trims", $10^{\text {th }}$ International LS_DYNA Users Conference, Detroit, 2008

[2] Alwerfalli, D., Lash, T., "Design For Six Sigma (DFSS) as a Proactive Business Process", Proceedings of the 2012 International Conference on Industrial Engineering and Operations Management. Istanbul, Turkey, July 3 - 6, 2012

[3] Yuin Wu, Alan Wu. "Taguchi Methods for Robust Design", ASME PRESS, 2000.

[4] Genichi Taguchi, Subir Chowdhury, Yuin Wu. "Taguchi's Quality Engineering”, ASI Consulting Group, LLC Livonia, Michigan. 